\title{
PSEUDO-ELLIPTIC INTEGRALS, UNITS, AND TORSION
}

\author{
FRANCESCO PAPPALARDI and ALFRED J. VAN DER POORTEN ${ }^{M}$
}

(Received 15 March 2004; revised 1 December 2004)

Communicated by W. W. L. Chen

\begin{abstract}
We remark on pseudo-elliptic integrals and on exceptional function fields, namely function fields defined over an infinite base field but nonetheless containing non-trivial units. Our emphasis is on some elementary criteria that must be satisfied by a squarefree polynomial $D(x)$ whose square root generates a quadratic function field with non-trivial unit. We detail the genus 1 case.
\end{abstract}

2000 Mathematics subject classification: primary $11 \mathrm{~J} 70,11 \mathrm{~A} 65,11 \mathrm{~J} 68$.

Keywords and phrases: quadratic function field of characteristic zero.

\section{Pseudo-elliptic integrals}

The surprising integral

$$
\begin{aligned}
& \int \frac{6 x d x}{\sqrt{x^{4}+4 x^{3}-6 x^{2}+4 x+1}} \\
& =\log \left(x^{6}+12 x^{5}+45 x^{4}+44 x^{3}-33 x^{2}+43\right. \\
& \left.\quad+\left(x^{4}+10 x^{3}+30 x^{2}+22 x-11\right) \sqrt{x^{4}+4 x^{3}-6 x^{2}+4 x+1}\right)
\end{aligned}
$$

is a nice example of a class of pseudo-elliptic integrals

$$
\int \frac{f(x) d x}{\sqrt{D(x)}}=\log (a(x)+b(x) \sqrt{D(x)}) .
$$

Here we take $D$ to be a monic polynomial defined over $\mathbb{Q}$, of even degree $2 g+2$, and not the square of a polynomial; $f, a$, and $b$ denote appropriate polynomials. We

This paper was constructed during a visit by the second author to Italy supported in part by a GNSAGA INDAM grant; his work is also supported by a grant from the Australian Research Council.

(C) 2005 Australian Mathematical Society 1446-7887/05 \$A2.00+0.00 
suppose $a$ to be nonzero, say of degree $m$ at least $g+1$. We will see that necessarily $\operatorname{deg} b=m-g-1$, that $\operatorname{deg} f=g$, and that $f$ has leading coefficient $m$. In our example, $m=6$ and $g=1$.

Plainly, if (1) holds then it remains true with $\sqrt{D}$ replaced by its conjugate $-\sqrt{D}$. Adding the two conjugate identities, we see that

$$
\int 0 d x=\log \left(a^{2}-D b^{2}\right)
$$

Thus $a^{2}-D b^{2}$ is some constant $k$, and must be nonzero because $D$ is not a square. In other words, $u=a+b \sqrt{D}$ is a nontrivial unit in the function field $\mathbb{Q}(x, \sqrt{D})$; and $\operatorname{deg} a=m$ implies $\operatorname{deg} b=m-g-1$ is immediate.

Differentiating (2) yields $2 a a^{\prime}-2 b b^{\prime} D-b^{2} D^{\prime}=0$. Hence $b \mid a a^{\prime}$, and since $a$ and $b$ must be relatively prime because $u$ is a unit, it follows that $b \mid a^{\prime}$. Set $f=a^{\prime} / b$, noting that indeed $\operatorname{deg} f=g$ and that $f$ has leading coefficient $m$ because $a$ and $b$ must have the same leading coefficient. That common coefficient is 1 without loss of generality since we may freely choose the constant produced by the indefinite integration.

Moreover,

$$
u^{\prime}=a^{\prime}+b^{\prime} \sqrt{D}+\frac{b D^{\prime}}{2 \sqrt{D}}=a^{\prime}+\frac{2 b b^{\prime} D+b^{2} D^{\prime}}{2 b \sqrt{D}}=a^{\prime}+\frac{a a^{\prime}}{b \sqrt{D}} .
$$

So, remarkably, $u^{\prime}=f(b \sqrt{D}+a) / \sqrt{D}=f u / \sqrt{D}$.

Thus, to verify (1) it suffices to make the not altogether obvious substitution $u(x)=a+b \sqrt{D}$, of course given that $u$ is a unit of the order $\mathbb{Q}[x, \sqrt{D}]$.

REMARK. The case $g=0$, say $D(x)=x^{2}+2 v x+w$, is useful for orienting oneself. Here $(x+v)+\sqrt{D}$ is a unit, of norm $v^{2}-w$, and indeed

$$
\int \frac{d x}{\sqrt{x^{2}+2 v x+w}}=\operatorname{arsinh} \frac{x+v}{\sqrt{w-v^{2}}}=\log \left(x+v+\sqrt{x^{2}+2 v x+w}\right) .
$$

Notice that $\operatorname{deg} f=0$ and has leading coefficient 1 , as predicted.

\section{Units in quadratic extension fields, and torsion}

2.1. Number fields Let $N$ be a positive integer, not a square, and set $\omega=\sqrt{N}$. It is easy to apply the Dirichlet box principle to prove that an order $\mathbb{Z}[\omega]$ of a quadratic number field $\mathbb{Q}(\omega)$ contains nontrivial units. Indeed, by that principle there are infinitely many pairs of integers $(p, q)$ so that $|q \omega-p|<1 / q$, whence $\left|p^{2}-N q^{2}\right|<$ $2 \sqrt{N}+1$. It follows, again by the box principle, that there is an integer $l$ with 
$0<|l|<2 \sqrt{N}+1$ so that the equation $p^{2}-N q^{2}=l$ has infinitely many pairs $(p, q)$ and $\left(p^{\prime}, q^{\prime}\right)$ of solutions with $p \equiv p^{\prime}$ and $q \equiv q^{\prime}(\bmod l)$. For each such distinct pair, $a l=p p^{\prime}-N q q^{\prime}, b l=p q^{\prime}-p^{\prime} q$, yields $a^{2}-N b^{2}=1$.

2.2. Function fields Just so, in the function field case already introduced, there are infinitely many pairs of polynomials $p(x)$ and $q(x)$ so that $\operatorname{deg}(q \sqrt{D}-p)<-\operatorname{deg} q$, whence $\operatorname{deg}\left(p^{2}-D q^{2}\right) \leq g$. But a second application of the box principle fails when the base field, $\mathbb{Q}$ in our introductory discussion, is infinite; because there are then infinitely many distinct polynomials of bounded degree. In that case, the existence of a nontrivial unit (thus, one not an element of the base field) is unusual happenstance. Accordingly, we say that a function field $\mathbb{Q}(x, \sqrt{D})$ with a nontrivial unit $a+b \sqrt{D}$ is an exceptional function field and we call $D$ an exceptional polynomial.

\subsection{Torsion on the Jacobian of a hyperelliptic curve A slight change of viewpoint,} emphasising the hyperelliptic curve $\mathscr{C}: y^{2}=D(x)$, may clarify matters. A function $u=a+b y$ is a unit precisely if its divisor is supported only at infinity. However, $\mathscr{C}$ has two points at infinity, say $O$ and $S$ (or $\infty_{-}$and $\infty_{+}$if one prefers) and so the divisor of $u$ is some multiple, say $m(S-O)$, of the divisor $S-O$ at infinity. Because $u$ is a function, this is to say that the class of $S-O$ on the Jacobian of $\mathscr{C}$ is torsion of order $m$. In the case $\operatorname{deg} D=4$, so genus $g=1$ if $D$ is squarefree, we may take $O$ as the zero of the elliptic curve $\mathscr{C}$ and report that the point $S$ on $\mathscr{C}$ is torsion of order $m=\operatorname{deg} a$.

\section{Exceptional quadratic fields}

It is appropriate to identify straightforward properties of the squarefree polynomial $D(x)=y^{2}$ sufficient or just necessary that the field $\mathbb{Q}(x, y)$ be exceptional.

Suppose, therefore, that $\mathbb{Q}(x, y)$ is exceptional, so that we have a unit $u=a+b y$ or, more helpfully, an identity $b^{2} D=a^{2}-k$ with $a, b \in \mathbb{Q}[x]$ and $k \in \mathbb{Q} \backslash\{0\}$. It will be helpful to set $k=c^{2}$. We note immediately that the two polynomials $a-c$ and $a+c$, which are conjugate over $\mathbb{Q}$ if $k$ is not a square, are relatively prime.

We have $b^{2} D=(a-c)(a+c)$. Hence if $k$ is not a square in $\mathbb{Q}, b$ must factor in $\mathbb{Q}(c)[x]$ as a norm $d \bar{d}$, where the overline - denotes conjugation in the quadratic extension $\mathbb{Q}(c)$, and $D$ factorises over $\mathbb{Q}(c)$ as the product of the polynomial $(a-c) / d^{2}$, and of its conjugate. In particular, $\operatorname{deg} b=m-g-1$ must be even.

If, however, $k$ is a square in $\mathbb{Q}$ (thus, in particular, always if $\operatorname{deg} b=m-g-1$ is odd) then we seem to see only that $b$ must have a factor $d$ defined over $\mathbb{Q}$ so that both $2 \operatorname{deg} d$ and $2 m-(2 g+2)-2 \operatorname{deg} d$ do not exceed $m=\operatorname{deg}(a-c)=\operatorname{deg} a$. That is, we have $m-(2 g+2) \leq 2 \operatorname{deg} d \leq m$. 
THEOREM 3.1. Set $y^{2}=D(x)$, with D monic, squarefree, and of degree $2 g+2$. Suppose the domain $\mathbb{Q}[x, y]$ contains a unit of degree $m>g$ and norm $k$.

(a) If $m$ and $g$ have the same parity then $k=c^{2}$ is a square.

(b) If $k=c^{2}$ with $c \in \mathbb{Q}$, there is a positive integer $s$ so that $D$ is a product of polynomials over $\mathbb{Q}$ of degrees $m-2 s$ and $2 g+2+2 s-m$. Thus $D$ is reducible over $\mathbb{Q}$ if $m$ is odd.

(c) If $k=c^{2}$ is not a square in $\mathbb{Q}$ then $D$ factorises over $\mathbb{Q}(c)$ as a product of two polynomials conjugate over $\mathbb{Q}(c)$, so each of degree $g+1$.

Note that the compactly written assertion (b) includes the possibility that $D$ is irreducible if $m$ is even, and (since both the stated degrees must be nonnegative) that it implicitly entails upper and lower bounds on the integer $s$. Assertion (c) implies that the Galois group of $D$ is restricted by \# Gal $(D) \mid 2((g+1) !)^{2}$. Thus, if $g=1$ it is the dihedral group on four elements or one of its subgroups.

We observe also that the statements of the theorem, which refer only to the polynomial $D$ and the torsion order $m$, do not include all the information that may be extracted from the remarks preceding the proclamation of the theorem.

REMARK. It should be no surprise that none of the criteria of the theorem suffices to guarantee obtaining an exceptional quadratic function field. We detail the case $g=1$ in Section 6 at page 342 below.

\section{Continued fractions}

4.1. Number fields There is a well-known algorithm in the number field case yielding the fundamental unit of the order $\mathbb{Z}[\sqrt{N}]$. As before set $\omega=\sqrt{N}$ and suppose $A$ is the integer part of $\omega$. The zero-th step in the continued fraction expansion of $\omega+A$ is

$$
\omega+A=2 A-(\bar{\omega}+A)
$$

and this and a typical consequent step is of the shape

$$
\left(\omega+P_{h}\right) / Q_{h}=a_{h}-\left(\bar{\omega}+P_{h+1}\right) / Q_{h} ; \quad \text { in brief } \omega_{h}=a_{h}-\bar{\rho}_{h} .
$$

Thus $P_{h}+P_{h+1}+(\omega+\bar{\omega})=a_{h} Q_{h}$, and because the next complete quotient $\omega_{h+1}$ is the reciprocal of the remainder $-\bar{\rho}_{h}$ we must also have $\left(\omega+P_{h+1}\right)\left(\bar{\omega}+P_{h+1}\right)=-Q_{h} Q_{h+1}$. In particular, certainly $Q_{h+1}$ divides the norm $\left(\omega+P_{h+1}\right)\left(\bar{\omega}+P_{h+1}\right)$.

Here the $P_{h}$ and $Q_{h}$ are integers, and it is readily shown they all satisfy

$$
0<2 P_{h}+(\omega+\bar{\omega})<\omega-\bar{\omega}, \quad 0<Q_{h}<\omega-\bar{\omega}
$$


proving, by the box principle, that the continued fraction expansion of $\omega$ is periodic. Moreover, one notices that always both

$$
\omega_{h}>1 \text { while }-1<\bar{\omega}_{h}<0 \text {, and } \rho_{h}>1 \text { while }-1<\bar{\rho}_{h}<0 \text {. }
$$

It follows that conjugation of the continued fraction tableau, replacing

$$
\omega_{h}=a_{h}-\bar{\rho}_{h} \quad \text { by } \quad \rho_{h}=a_{h}-\bar{\omega}_{h} \text {, }
$$

again gives a continued fraction expansion - in particular, $a_{h}$ which began life as the integer part of $\omega_{h}$, also is the integer part of $\rho_{h}$-reversing the order of the lines of the original expansion. Because line zero (3) is symmetric it occurs in the expansion of $\rho_{h}$, and because the expansion of $\omega+A$ is periodic it follows that it is in fact purely periodic, moreover with a symmetry: if the period length is $r$ then the word $a_{1}, a_{2}, \ldots, a_{r-1}$ must be a palindrome.

One obtains the fundamental unit $a+b \omega$ by computing the convergent

$$
\left[A, a_{1}, a_{2}, \ldots, a_{r-1}\right]=a / b
$$

4.2. Function fields Mutatis mutandis, the function field argument is identical. We set $y^{2}=D(x)$ as before. Plainly we may write $D$ as $D=A^{2}+R$, where $\operatorname{deg} A=g+1$ and $\operatorname{deg} R<g$; then $A$ is the polynomial part of the Laurent series $y \in \mathbb{Q}\left(\left(x^{-1}\right)\right)$. We expand $y+A$ in complete analogy with the numerical case, but now selecting the partial quotients $a_{h}$ as the polynomial part of the respective complete quotients $y_{h}:=\left(y+P_{h}\right) / Q_{h}$. The bounds (4) become

$$
\operatorname{deg} P_{h}=g+1 \quad \text { and } \quad \operatorname{deg} Q_{h} \leq g
$$

and of course do not guarantee periodicity, because the base field $\mathbb{Q}$ is infinite. The conditions (5) for reduction turn into

$$
\begin{aligned}
\operatorname{deg}\left(y+P_{h}\right)>\operatorname{deg} Q_{h} & \text { but } & \operatorname{deg}\left(\bar{y}+P_{h}\right)<\operatorname{deg} Q_{h} \quad \text { and therefore } \\
\operatorname{deg}\left(y+P_{h+1}\right)>\operatorname{deg} Q_{h} & \text { but } & \operatorname{deg}\left(\bar{y}+P_{h+1}\right)<\operatorname{deg} Q_{h} .
\end{aligned}
$$

As in the number field case, conjugation reverses the continued fraction tableaux. Thus, if the expansion of $y+A$ happens to be periodic then it has the symmetries of the number field case and the continued fraction expansion yields a unit of norm 1 , given by the convergent (6). Note that in the function field case there is the possibility of quasi-periodicity $a_{h+r}=c_{h} a_{h}$, non-zero constants $c_{h}$, see [19], rather than periodicity proper: $a_{h+r}=a_{h}$. 
4.3. Quasi-periodicity Suppose now that $D$ is exceptional in that the function field $\mathbb{Q}(x, y)$ contains a unit $u$, of norm $-\kappa$. By general principles that entails that some $Q_{i}$ is $\pm \kappa$, say $Q_{r}=\kappa$ with $r$ odd. That is, line $r$ of the continued fraction expansion of $y+A$ is

line $r$ :

$$
y_{r}:=(y+A) / \kappa=2 A / \kappa-(\bar{y}+A) / \kappa ;
$$

here we have used $\left(5^{\prime}\right)$ to deduce that necessarily $P_{r}=P_{r+1}=A$. We recall that line 0:

$$
y+A=2 A-(\bar{y}+A) .
$$

By conjugation of the $(r+1)$-line tableau showing that $y+A$ is quasi-periodic we see immediately also that

line $2 r$ :

$$
y_{2 r}:=y+A=2 A-(\bar{y}+A),
$$

so that in any case if $y+A$ has a quasi-periodic continued fraction expansion then it is periodic of period twice the quasi-period. This is a result of Berry [3]; it applies to arbitrary quadratic irrational functions whose trace is a polynomial. Other elements $(y+P) / Q$ of $\mathbb{Q}(x, y)$, with $Q$ dividing the norm $(y+P)(\bar{y}+P)$, may be honestto-goodness quasi-periodic, that is, not also periodic. If $y$ has trace $t$, rather than zero trace, replace line zero of the expansion by $y+A-t=2 A-t-(\bar{y}+A-t)$ and so on in the story just told. To be able to do that $t$ should of course be 'integral', that is, a polynomial.

Further, if $\kappa \neq-1$ then $r$ must be odd. To see that, notice the identity

$$
B\left[C a_{0}, B a_{1}, C a_{2}, B a_{3}, \ldots\right]=C\left[B a_{0}, C a_{1}, B a_{2}, C a_{3}, \ldots\right],
$$

reminding one how one multiplies a continued fraction expansion by some quantity; this cute formulation of the multiplication rule is due to Schmidt [15]. The 'twisted symmetry' occasioned by division by $\kappa$, equivalent to the existence of a non-trivial quasi-period, is noted by Christian Friesen [7].

In summary: if quasi-periodic it is periodic, and then the continued fraction expansion of $y=\sqrt{D(x)}$ has the symmetries of the more familiar number field case, as well as the twisted symmetries occasioned by a nontrivial $\kappa$.

REMARK. The conclusion just stated is surely well known. Certainly it is asserted by Adams and Razar [1], but without the couple of lines of argument we add here. The second of us is indebted to notes of Street [16], and related enquiries from Brian Conrad, for being reminded of this unneeded gap in the literature and of the desirability of detailing a straightforward argument. A much clumsier version of the story told here is given in [19], however with additional introductory details that may be helpful to the reader. 
THEOREM 4.1. Set $\mathscr{C}: y^{2}=D(x)$, with $D$ monic, squarefree, and of degree $2 g+2$. Suppose the divisor at infinity on the Jacobian of the curve $\mathscr{C}$ is torsion of order $m>1$, equivalently the domain $\mathbb{Q}[x, y]$ is exceptional in containing nontrivial units, and its fundamental unit $u=a+b y$ is of degree $m$, and say of norm $k$. Denote the continued fraction expansion of $y$ by $y=\left[A, a_{1}, a_{2}, a_{3}, \ldots\right]$. Then, further to Theorem 3.1,

(a) if $\left[A, a_{1}, a_{2}, \ldots, a_{r-1}\right]=a / b$ with $r$ even, then $k=1$;

(b) if $k=c^{2}$ with $c \in \mathbb{Q}$, then the polynomial b factorises over $\mathbb{Q}$ as say $b=d_{+} d_{-}$, and $D$ is reducible over $\mathbb{Q}$ because it factorises as the product of the nontrivial polynomials $(a+c) / d_{+}^{2}$ and $(a-c) / d_{-}^{2}$;

(c) if $k=c^{2}$ is not a square in $\mathbb{Q}$ then the polynomial b factorises over $\mathbb{Q}(c)$ as a product $b=d \bar{d}$ of polynomials conjugate over $\mathbb{Q}(c)$, and $D$ factorises over $\mathbb{Q}(c)$ as a product of the two polynomials $(a+c) / d^{2}$ and $(a+\bar{c}) / \bar{d}^{2}$.

For $g=1$, we must have $m=r+1$ by the bounds (4'), so the parities of $m$ and $r$ are of course different; in particular, $m$ odd entails the norm $k=1$. One readily notices that symmetry implies that always if $r$ is odd the parities of $m$ and $g$ are different; the converse is not true if $g>1$. For the rest, Theorem 4.1 fills in details omitted from Theorem 3.1.

An important such 'detail', is the observation that if, say, $2 \operatorname{deg} d_{+}=m$ so $d_{+}^{2}=$ $a+c$, then $D d_{-}^{2}=a-c=d_{+}^{2}-2 c$. So also $d_{+}+y d_{-}$is a unit of $\mathbb{Q}[x, y]$ plainly contradicting the minimality of $m$, that is, that $u$ is a fundamental unit.

Furthermore, we see that $D$ has a factor of degree at most $g$ if the period length $r=2 h$ is even. For then, by conjugation, the line

$$
\left(y+P_{h}\right) / Q_{h}=a_{h}-\left(\bar{y}+P_{h+1}\right) / Q_{h}
$$

is symmetric, that is $P_{h+1}=P_{h}$, and so $Q_{h}$ divides $P_{h}$. But then $Q_{h}$ also divides the norm $\left(y+P_{h}\right)\left(\bar{y}+P_{h}\right)$ and that entails $Q_{h}$ is a factor of $D$.

There are contexts in which one would like to be certain that a polynomial $D$ is not exceptional. Our results have the following consequence.

COROLlaRY 4.2. If a monic polynomial $D$ of even degree at least 4 is irreducible and with Galois group the full symmetric group then $D$ is not exceptional; that is, the continued fraction expansion of $\sqrt{D}$ is not periodic.

\section{Exceptional polynomials}

In practice, the start of the continued fraction expansion of $y=\sqrt{D}$ quickly reveals whether or not $D$ is exceptional. For example, it is shown in [1] for $g=1$ that in $y_{h}=\left(y+P_{h}\right) / Q_{h}$ the divisor of $Q_{h}$ is $h+1$ times the divisor at infinity. Thus, by well 
known properties of Neron-Tate height, the number of decimal digits of the numerators and denominators of the coefficients of $Q_{h}$ (and then also of $P_{h}$ ) is $O\left(h^{2}\right)$ unless the divisor at infinity is torsion. Moreover, in practice that explosion in complexity of $Q_{h}$ is immediately evident; see [17] for an example. Moreover, that same explosion in complexity occurs for arbitrary $g>0$ since it follows from addition on the Jacobian of the curve $y^{2}=D(x)$ being given by composition of quadratic forms, that is, by the continued fraction expansion of $y$; [5] or [11] explain this connection. In any case, [4], the matter of explosion of complexity of Padé approximants of algebraic functions of positive genus is far more general yet.

In the number field case, the fundamental unit of an order $\mathbb{Z}[f \omega]$ is some power of the fundamental unit of the domain of all integers of $\mathbb{Q}(\omega)$. For function fields over a base field of characteristic zero, however, an order $\mathbb{Q}[x, f(x) y]$ need not possess a unit at all, notwithstanding that $D=y^{2}$ be exceptional. In other words, periodicity of $y$ does not at all guarantee quasi-periodicity of $f y$ for a polynomial $f$ of positive degree. The requirement in our theorems that $D$ be squarefree thus really does matter. Specifically, although the continued fraction expansion is trivially quasi-periodic for $\operatorname{deg} D=2$, thus $y^{2}=D$ of genus $g=0$, this may not hold for $y^{2}=f^{2} D$, even though that curve is of genus 0 . There are interesting papers, see [9] and its references, discussing this issue.

\section{The quartic case}

The case $g=1$ is completely known over $\mathbb{Q}$, see [18] and its references, or for example [2]. In particular, one knows by Mazur's Theorem [13] that the only possibilities for $m$ are $m=2,3, \ldots, 10$, and 12. From [20] one learns that in the cases $m=10$ and $m=12$ it happens that in fact $k=c^{2}$ never is the square of a rational; that is, then $c$ is never rational.

For torsion $m \geq 4$, one may take $D_{m}(x)$ as $\left(x^{2}+v-w^{2}\right)^{2}+4 v(x+w)$ without loss of generality; $D_{3}(x)=\left(x^{2}-w^{2}\right)^{2}+4 v(x+w)$, while $D_{2}(x)=\left(x^{2}+u\right)^{2}+4 w$. Here $u, v$ and $w$ are rational parameters. For each $m=4,5, \ldots, 10,12$ these parameters are rational functions, detailed in [20], in a single rational parameter $t$.

THEOREM 6.1. Set $\mathscr{C}_{m}: y^{2}=D_{m}(x ; t)$, with $D_{m}$ monic, squarefree, and of degree 4 . Suppose the divisor at infinity on the Jacobian of the curve $\mathscr{C}_{m}$ is torsion of exact order $m>3$. Then $D_{m}(x ; t)$ is reducible over $\mathbb{Q}$ if $m$ is odd or in the cases listed in Table 2. Otherwise, its Galois group is the dihedral group $\mathscr{D}_{4}$, other than for the exceptions listed in Table 1.

PROOF. We know from the preceding theorems that $D_{m}(x, t)$ is reducible if $m$ is odd or if the norm $k_{m}(t)$ of the fundamental unit happens anyhow to be a square. 
Specifically, [20] reports that $k_{8}(t)=4(t-1)(2 t-1)^{2} / t^{3}, k_{6}(t)=4 t$, and $k_{4}(t)=4 t$, explaining several of the entries in Table 2. Thus we may suppose that $k=c^{2}$ with $c$ quadratic irrational over $\mathbb{Q}$.

The Galois group $G_{D}$ of $D=D_{m}$ is the dihedral group $\mathscr{D}_{4}$ exactly when the zeros of $D$ are $\alpha_{1}, \alpha_{3}, \alpha_{2}=\bar{\alpha}_{3}$, and $\alpha_{4}=\bar{\alpha}_{1}$, where ${ }^{-}=(14)(23)$ is conjugation over $\mathbb{Q}(c)$. Then $G_{D}$ is generated by that conjugation and $\sigma=(1234)$.

Conversely, given that $D$ factorises over $\mathbb{Q}(c)$, the cubic resolvent $C_{D}$ of $D$ must have a rational zero $\alpha_{1} \alpha_{3}+\alpha_{2} \alpha_{4}$. The other two zeros $\alpha_{1} \alpha_{2}+\alpha_{3} \alpha_{4}$ and $\alpha_{1} \alpha_{4}+\alpha_{2} \alpha_{3}$ are invariant under the conjugation but are transposed by $\sigma$ and, for that matter, also by the 4-cycle $\tau=(1243)$.

If these other zeros of $C_{D}$ are rational then both $\sigma$ and $\tau$ must be involutions commuting with the conjugation. Then, recalling that $D$ is irreducible over $\mathbb{Q}$, its Galois group $G_{D}$ is the Viergruppe $\mathscr{V}$. If the pair of zeros is irrational but $D$ factorises over the splitting field of $C_{D}$ then $\tau$ generates $G_{D}$ and the Galois group of $D$ is the cyclic group $\mathscr{C}_{4}$. Incidentally, we use the helpful remarks [10, Algorithm 4.2 on page 10], explicitly to distinguish the case $\mathscr{C}_{4}$ from $\mathscr{D}_{4}$.

Even calculations We investigate each case $m=12,10,8,6,4$ in detail using the data listed in [20]. For example, the cases $m=12$ and $m=10$ are given by

$$
\begin{aligned}
v_{12}(t) & =(t-1)(2 t-1)\left(3 t^{2}-3 t+1\right)\left(2 t^{2}-2 t+1\right) / t^{4} ; \\
w_{12}(t) & =-\left(6 t^{4}-16 t^{3}+14 t^{2}-6 t+1\right) / 2 t^{3} ; \\
v_{10}(t) & =\frac{t^{3}(2 t-1)(t-1)}{\left(t^{2}-3 t+1\right)^{2}} ; \quad w_{10}(t)=\frac{2 t^{3}-2 t^{2}-2 t+1}{2\left(t^{2}-3 t+1\right)} .
\end{aligned}
$$

Here the parameter $t$ runs through all 'regular' elements of $\mathbb{Q}$; in both cases the irregular rational values are $t=1, t=1 / 2$, and $t=0$.

By Theorem 3.1 (c) we know that $D_{m}(x ; t)$ factorises over $\mathbb{Q}(c)$; here of course $c=c(t)$ depends the rational parameter $t$. If $D_{m}(x ; t)$ also factorises over $\mathbb{Q}$ it must do so as a product $\left(x^{2}-p x+q\right)\left(x^{2}+p x+q^{\prime}\right)$. One solves (rather, Maple [12] solves) this condition for $p=p(t)$, in each case obtaining two polynomial equations in $p$ and $t$, with one an elliptic curve and the other a quadratic in an auxiliary variable. The condition that its discriminant be a square also is an elliptic curve.

In the case $m=12$, both of these equations ultimately transform birationally (here PARI-GP [14] lends a hand) to the minimal model $y^{2}=x^{3}-x^{2}+x$. This is is 24A4 in Cremona's tables [6]; thus with conductor 24. It has rank 0 and cyclic torsion of order 4 ; the torsion points are $(0,0),(1,1),(1,-1)$, and $\infty$ and correspond to irregular values of $t$. So $D_{12}(x ; t)$ is irreducible over $\mathbb{Q}$ for all regular $t \in \mathbb{Q}$.

When, instead, we check the cubic resolvent, for example when $m=10$, we find that its rational zero is $\left(2 t^{3}-4 t^{2}+4 t-1\right)\left(2 t^{3}-4 t^{2}+1\right) / 2\left(t^{2}-3 t+1\right)^{2}$ and if the discriminant of the remaining quadratic factor of $C_{D}$ is a square then the elliptic curve 
$s^{2}=\left(4 t^{2}-2 t-1\right)(2 t-1)$ must have admissible rational points. However, its minimal model $y^{2}=x^{3}+x^{2}-x$ is 20A2 in Cremona's tables and it has rank 0 and cyclic torsion of order 6 . The torsion points are $(0,0),( \pm 1, \pm 1)$, and $\infty$ and correspond to irregular values of $t$.

Following both the alternative approaches for each of $m=12$ and $m=10$ verifies a result we have used above, to wit Tran's result [20, page 400ff] that neither $\kappa_{12}(t)$ nor $\kappa_{10}(t)$ - see Section 4.3 on page 340 above - can be the square of a rational for regular $t \in \mathbb{Q}$.

For these and the remaining even cases $m=8, m=6$, and $m=4$, where we know that $k=\kappa_{m}(t)$ may be a square for some regular $t$, we followed both approaches and found that when $D_{m}(x ; t)$ is irreducible its Galois group $G_{D}$ is the dihedral group $\mathscr{D}_{4}$ except in the cases encapsulated in the following table.

TABLE 1.

\begin{tabular}{|c|c|c|c|}
\hline$m$ & $(v, w)$ & $G_{D}=\mathscr{C}_{2} \times \mathscr{C}_{2}$ & $G_{D}=\mathscr{C}_{4}$ \\
\hline 4 & $(t, 1 / 2)$ & $t=\frac{1}{16}\left(s^{2}-1\right)$ & $t=-\frac{1}{16} /\left(s^{2}+1\right)$ \\
6 & $(t(t-1), 1-t / 2)$ & $t=8 /\left(9-s^{2}\right)$ & - \\
8 & $\left((t-1)(2 t-1),-\left(2 t^{2}-4 t+1\right) / 2 t\right)$ & - & - \\
10 & $\left(t^{3}(2 t-1)(t-1) /\left(t^{2}-3 t+1\right)^{2}\right.$, & - & $*$ \\
& $\left.2 t^{3}-2 t^{2}-2 t+1 / 2\left(t^{2}-3 t+1\right)\right)$ & & \\
12 & $\left((t-1)(2 t-1)\left(3 t^{2}-3 t+1\right)\left(2 t^{2}-2 t+1\right) / t^{4}\right.$, & - & \\
& $\left.-\left(6 t^{4}-16 t^{3}+14 t^{2}-6 t+1\right) / 2 t^{3}\right)$ & & \\
\hline
\end{tabular}

Moreover, for $m$ even, $D_{m}(x, t)$ is irreducible except in the following cases:

TABLE 2.

\begin{tabular}{|c|c|c|c|c|}
\hline$m$ & $(v, w)$ & $D=f_{1} f_{2}$ & $D=f_{1} f_{2} f_{3}$ & $D=f_{1} f_{2} f_{3} f_{4}$ \\
\hline 4 & $(t, 1 / 2)$ & $t=\left\{\begin{array}{l}-s^{2}, \\
4 s^{4}-s^{2}\end{array}\right.$ & $t=-\left(\frac{s^{2}-1}{4}\right)^{2}$ & $t=-\left(\frac{s^{3}-s}{\left(s^{2}+1\right)^{2}}\right)^{2}$ \\
6 & $(t(t-1), 1-t / 2)$ & $t=\left\{\begin{array}{l}1-s^{2} \\
\left(1+s^{2}\right)^{2} \\
3 s^{2}+1\end{array}\right.$ & $t=1-\left(\frac{s^{2}-1}{s^{2}+3}\right)^{2}$ & - \\
8 & $\begin{array}{c}((t-1)(2 t-1), \\
\left.-\left(2 t^{2}-4 t+1\right) / 2 t\right)\end{array}$ & $t=1 /\left(s^{2}+1\right)$ & & \\
\hline
\end{tabular}

The notes $*$ and $\dagger$ refer to two special cases we resolved not to attempt to resolve. We found that rational points $(t, u)$ on the curve

$$
u^{2}=(t-1)\left(4 t^{2}-2 x-1\right)(2 t-1)\left(t^{2}-3 t+1\right) t
$$


give rise to cases $D_{10}(x ; t)$ with Galois group $\mathscr{D}_{4}$; and rational points on the curve

$$
u^{2}=\left(t^{4}-1\right)\left(t^{2}+2 t-1\right)
$$

give cases where $D_{8}(x ; t)$ splits into three factors over $\mathbb{Q}$. We expect that neither curve provides regular rational such $t$.

We leave the degenerate case $m=2$, where $D(x ; u, k)=\left(x^{2}+u\right)^{2}-k$, as an easy exercise.

Odd remarks In the odd cases $m=9, m=7$, and $m=5$, the final remark following Theorem 4.1 at page 341 , together with the detailed continued fraction expansions in [20], shows that

$$
\begin{array}{rr}
\left(x-\frac{1}{2}\left(t^{3}-3 t^{2}+4 t-1\right)\right) & \text { divides } D_{9}(x ; t), \\
\left(x+\frac{1}{2}\left(t^{2}-3 t+1\right)\right) & \text { divides } D_{7}(x ; t), \\
\left(x-\frac{1}{2}(t+1)\right) & \text { divides } D_{5}(x ; t) ;
\end{array}
$$

here

$$
\begin{array}{lll}
v_{9}(t)=t^{2}(t-1)\left(t^{2}-t+1\right), & w_{9}(t)=-\frac{1}{2}\left(t^{3}-t^{2}-1\right), & t \in \mathbb{Q} \backslash\{0,1\}, \\
v_{7}(t)=t^{2}(t-1), & w_{7}(t)=-\frac{1}{2}\left(t^{2}-t-1\right), & t \in \mathbb{Q} \backslash\{0,1\}, \\
v_{5}(t)=t, & w_{5}(t)=-\frac{1}{2}(t-1), & t \in \mathbb{Q} \backslash\{0\} .
\end{array}
$$

As always, the data (from [20]) must be used modulo typos. Worse, the notation of [20] is slightly different from that of here and in [18]; its $v$ is our $4 v$.

For completeness we remark that in these cases the residual cubic factor $G_{m}(x ; t)$ is reducible in the case $m=5$ and $t=s^{2}(s+1) /(s+1)$ and that then the surviving quadratic factor is irreducible. With finitely many possible exceptions, namely unlikely rational points on certain curves of genus more than 1, the Galois groups of the irreducible $G_{m}(x ; t)$ is always $\mathscr{S}_{3}$.

Specifically, the respective discriminants $F_{m}(t)$ of the cubic factors are

$$
\begin{aligned}
& F_{7}(t)=t(t-1)\left(t^{3}-8 t^{2}+5 t+1\right), \\
& F_{9}(t)=t(t-1)\left(t^{2}-t+1\right)\left(t^{3}-6 t^{2}+3 t+1\right), \quad \text { and } \\
& F_{5}(t)=t(t-1)\left(t^{3}-8 t^{2}+5 t+1\right)
\end{aligned}
$$

The last case is Cremona's curve 20A2, which has rank 0 and torsion 2 . We saw that $G_{7}(x ; t)$ is irreducible because a putative rational zero corresponds to a rational point on the curve 14A4 with rank 0 and torsion 2 . We found a complicated genus 2 curve not warranting report whose rational points might allow $G_{9}(x ; t)$ to factorise. 
The case $m=3$ is degenerate; however, plainly

$$
\begin{aligned}
D_{3}(x ; v, w) & =\left(x^{2}-w^{2}\right)^{2}+4 v(x+w) \\
& =(x+w)\left(x^{3}-w x^{2}-w^{2} x-4 v+w^{3}\right)=:(x+w) F .
\end{aligned}
$$

If $F$ is irreducible, then its Galois group is $\mathscr{A}_{3}$ if and only if $v=8 t^{2} w^{3} /\left(27 t^{2}+1\right)$. Further, $F$ has a zero $r$ when $v=(w+r)(w-r)^{2} / 4$; specifically

$$
F=(x-r)\left(x^{2}-(w-r) x-w^{2}-r w+r^{2}\right) .
$$

$F$ splits as the product of three linear factors when $v=8 w^{3}\left(s^{2}-1\right)^{2} /\left(\left(s^{2}+3\right)^{3}\right)$. The reader may find it a useful exercise to extract other details.

\section{References}

[1] W. W. Adams and M. J. Razar, 'Multiples of points on elliptic curves and continued fractions'. Proc. London Math. Soc. 41 (1980), 481-498.

[2] R. M. Avanzi and U. M. Zannier, 'Genus one curves defined by separated variable polynomials and a polynomial pell equation', Acta Arith. 99 (2001), 227-256.

[3] T. G. Berry, 'On periodicity of continued fractions in hyperelliptic function fields', Arch. Math. 55 (1990), 259-266.

[4] E. Bombieri and P. B. Cohen, 'Siegel's lemma, Padé approximations and Jacobians', Ann. Scuola Norm. Sup. Pisa Cl. Sci. (4) 25 (1997), 155-178.

[5] D. G. Cantor, 'Computing in the Jacobian of a hyperelliptic curve', Math. Comp. 48 (1987), 95-101.

[6] J. E. Cremona, Algorithms for modular elliptic curves (Cambridge Univ. Press, 1997). Available on-line at http://www.maths.nott.ac.uk/personal/jec/book/amec.html

[7] C. Friesen, 'Continued fraction characterization and generic ideals', in: The arithmetic of function fields (Columbus, OH, 1991) (eds. D. Goss, D. R. Hayes and M. I. Rosen), Ohio State Univ. Math. Res. Inst. Publ. 2 (Walter de Gruyter, Berlin, 1992) pp. 465-474.

[8] D. Goss, D. R. Hayes and M. I. Rosen (eds.), The arithmetic of function fields (Columbus, OH, 1991), Ohio State Univ. Math. Res. Inst. Publ. 2 (Walter de Gruyter, Berlin, 1992).

[9] I. Hardy, Y. Hellegouarch and R. Paysant-Le-Roux, 'Fractions continues normales dans un corps de fonctions hyperelliptiques', Acta Arith. 101 (2002), 19-37.

[10] A. D. Healy, 'Resultants, resolvents, and computation of Galois groups', available on-line at hitp://www.alexhealy.net/papers/math250a.pdf.

[11] K. E. Lauter, 'The equivalence of the geometric and algebraic group laws for jacobians of genus 2 curves', in: Proceedings of the conferences in memory of Ruth Michler, AMS Contemp. Math. Series (Amer. Math. Soc., Providence, RI, to appear).

[12] Maple, http://www.maplesoft.com/

[13] B. Mazur, 'Modular curves and the Eisenstein ideal', Inst. Hautes Études Sci. Publ. Math. 47 (1977), 33-186.

[14] PARI-GP, see http://pari.math.u-bordeaux.fr

[15] W. M. Schmidt, 'On continued fractions and diophantine approximation in power series fields', Acta Arith. 9 (2000), 139-166. 
[16] E. Street, 'Pell's equation and Laurent fields', manuscript, 2003.

[17] A. J. van der Poorten, 'Non-periodic continued fractions in hyperelliptic function fields', Bull. Austral. Math. Soc. 64 (2001), 331-343.

[18] - 'Periodic continued fractions and elliptic curves', in: High primes and misdemeanours: lectures in honour of the 60th birthday of Hugh Cowie Williams (eds. A. van der Poorten and A. Stein), Fields Institute Communications Series (Amer. Math. Soc., Providence, RI, 2004) pp. 353-365.

[19] A. J. van der Poorten and X. C. Tran, 'Quasi-elliptic integrals and periodic continued fractions', Monatshefie Math. 131 (2000), 155-169.

[20] —_, 'Periodic continued fractions in elliptic function fields', in: Algorithmic number theory (Proc. Fifth International Symposium, ANTS-V, Sydney, NSW, Australia July 2002) (eds. C. Fieker and D. R. Kohel), Lecture Notes in Comput. Sci. 2369 (Springer, Berlin, 2002) pp. 390-404.

Dipartimento di Matematica

Università degli Studi Roma Tre

L.go San Leonardo Murialdo, 1

I-00146 Roma

Italy

e-mail: pappa@mat.uniroma3.it
Centre for Number Theory Research

1 Bimbil Place, Killara

Sydney NSW 2071

Australia

e-mail: alf@math.mq.edu.au 